\title{
Stakeholders, actores estratégicos en la construcción de marca*
}

\author{
Jaime Eduardo Alzate Sanz ${ }^{* *}$ \\ Jaime Alberto Orozco Toro***
}

Recibido: 2017-06-28 • Enviado a pares: 2017-09-06

Aprobado por pares: 2017-10-17 • Aceptado: 2017-12-11

DOI: 10.22395/angr.v16n32a6

\begin{abstract}
Resumen
Los stakeholders, o grupos de interés, son cada día más importantes para la construcción de marca, especialmente por la necesidad de generar una comunicación diferenciada de parte de la empresa, la cual debe demostrar una identidad sólida, que genere procesos de creación de una correcta imagen y una reputación corporativa fuerte. En este aspecto, este artículo responde a una necesidad prioritaria: conocer los grupos de interés para la creación de estrategias de construcción de marca, incluso a partir de herramientas de actualidad como el marketing experiencial. Para esto se realizó una revisión teórica de autores reconocidos en el campo de la marca, la comunicación corporativa y el marketing, a través de bases de datos especializadas, donde se tuvieron en cuenta textos académicos, investigaciones y artículos científicos. Luego de tener estos insumos, se desarrollaron temáticas relevantes para el estudio de los stakeholders y su relación con la comunicación de las empresas. Esto permitió realizar un análisis detallado de los intangibles de la empresa, lo que aporta a la generación de conocimiento útil para el área del branding. Las principales conclusiones giran en torno a la relevancia que tienen los stakeholders en la gestión de la identidad de la marca, con miras a la generación de una correcta imagen y reputación corporativa. Este asunto es clave en la comunicación de cualquier empresa, debido a que en la actualidad el manejo adecuado de los intangibles es la clave del éxito empresarial y de un buen vínculo con los diferentes stakeholders.
\end{abstract}

Palabras clave: stakeholders; identidad; imagen; reputación corporativa; marca; posicionamiento; marketing; empresa.

Este artículo es resultado del proyecto de investigación 'Branding' corporativo en empresas de Manizales, financiado por la Vicerrectoría de Investigaciones y Postgrados de la Universidad de Caldas (Colombia). Además, hace parte de la tesis doctoral (por ahora con el mismo nombre de la investigación mencionada) que se está desarrollando en el Doctorado en Diseño y Creación de la misma universidad, con la tutoría del coautor.

* Candidato a doctor en Diseño y Creación. Magíster en Diseño y Creación Interactiva. Profesor-investigador Universidad de Caldas (Colombia). jaime.alzate@ucaldas.edu.co. https://orcid.org/0000-0002-1613-9117

... Doctor en Medios, Comunicación y Cultura. Investigador del grupo Epilión de la Universidad Pontificia Bolivariana (Colombia). jaime.orozco@upb.edu.co. https://orcid.org/0000-0002-8152-7474 


\title{
Stakeholders: Strategic Actors in Brand Building
}

\begin{abstract}
The stakeholders, also known as groups of interest, are increasingly important for brand building, especially because of the need to generate differentiated communication on the part of the company, which must demonstrate a solid identity that generates processes for the creation of a correct image and a strong corporate reputation. In this aspect, this article meets a priority need: to know the groups of interest for the creation of brand building strategies, even from current tools such as experiential marketing. For this, a theoretical review of recognized authors in the field of branding, corporate communication, and marketing was carried out through specialized databases: academic texts, research, and scientific articles were taken into account. After having these inputs, relevant topics for the study of the stakeholders and their relationship with the communication of the companies were developed. This allowed for a detailed analysis of the company's intangibles, which contributes to the generation of useful knowledge for the field of branding. The main conclusions revolve around the relevance that stakeholders have in the management of the identity of the brand, with a view to generating a correct image and corporate reputation. This issue is key in the communication of any company, because currently the proper management of intangibles is the key to business success, and a good link with different stakeholders.
\end{abstract}

Keywords: Stakeholders; identity; image; corporate reputation; brand; positioning; marketing; company.

\section{Stakeholders, atores estratégicos na construção de marca}

\begin{abstract}
Resumo
Os stakeholders ou grupos de interesse são cada dia mais importantes para construir uma marca, em especial, devido à necessidade de a empresa gerar uma comunicação diferenciada, a qual deve demonstrar uma identidade sólida, que gere processos de criação de uma correta imagem e de uma reputação corporativa forte. Nesse aspecto, este artigo responde a uma necessidade prioritária: conhecer os grupos de interesse para criar estratégias de construção de marca, inclusive a partir de ferramentas de atualidade como o marketing experiencial. Para isso, foi realizada uma revisão teórica de autores reconhecidos no campo da marca, da comunicação corporativa e do marketing por meio de bases de dados especializadas, em que foram considerados textos acadêmicos, pesquisas e artigos científicos. Em seguida, foram desenvolvidas temáticas relevantes para o estudo dos stakeholders e sua relação com a comunicação das empresas. Isso permitiu realizar uma análise detalhada dos intangíveis da empresa, o que contribui para gerar conhecimento útil para a área de branding. As principais conclusões giram em torno da relevância que os stakeholders têm na gestão da identidade da marca, com vistas à geração de uma correta imagem e reputação corporativa. Esse assunto é fundamental na comunicação de qualquer empresa devido a que, na atualidade, a gestão adequada dos intangíveis é a chave do sucesso empresarial e de um bom vínculo com os diferentes stakeholders.
\end{abstract}

Palavras-chave: stakeholders; identidade; imagem; reputação corporativa; marca; posicionamento; marketing; empresa. 


\section{Introducción}

Para una empresa, institución u organización la marca se ha convertido en su valor más importante, pues a través de esta es posible generar una relación de confianza con sus stakeholders o públicos que son afectados o afectan el desarrollo de una empresa (Freeman, Harrison, Wicks, Parmar \& Colle, 2010). Este clima de confianza se establece a través de la comunicación de marca, con miras a la generación de imagen y reputación, a partir de la generación de una identidad de marca. En esta línea se puede considerar que "la marca es el referente visual de la identidad de una empresa, institución, organización, producto, servicio, etc." (Costa, 2004, p. 99). La marca, además de posibilitar la comunicación de cualquier organización en diversos espacios, ofrece otros valores que van más allá del simple nombre. Para García (2005) el concepto de marca, además de abordar aspectos identitarios, tiene que ver con los aspectos legales que representan una organización. Por esta razón, desde el punto de vista legal, la marca es similar a la identificación de cualquier ciudadano, es única e irreemplazable, protegida y reconocida tanto por la ley como por los diferentes sectores sociales. Sin embargo, este intangible debe proyectarse de forma adecuada, para que no se generen conceptos negativos en la estructura de comunicación interna y externa.

Según estas condiciones, la marca estará conformada por diferentes elementos tangibles e intangibles, es decir, los productos y el conjunto de símbolos que los representan. Estos elementos deben organizarse coherentemente en una estructura de comunicación, con el fin de encontrar unidad y fuerza en la marca, para que de esta forma se pueda proyectar confianza y solidez.

Los intangibles deben estar conectados con los grupos de interés, los cuales son la razón de ser de cualquier empresa. Hoy en día no es posible que una empresa pueda proyectarse en el mercado si no posee el respaldo de los stakeholders.

\section{Metodología}

Este artículo fue desarrollado a partir de la revisión y análisis de diversas teorías de autores que abordan los estudios sobre la marca desde diferentes perspectivas. Se tuvieron en cuenta teorías y conceptos relacionados con los grupos de interés o stakeholders, con el fin de conectarlos estrechamente con los intangibles de la marca, para de esta forma construir un cuerpo teórico útil para el campo del branding. Luego de estas conexiones e interrelaciones de temáticas se llegó a ciertas conclusiones, las cuales son parte de los resultados parciales de la investigación "Branding corporativo en empresas de Manizales". Este insumo es importante para el desarrollo de la tesis doctoral, que por el momento tiene el mismo nombre de la investigación mencionada. Se pretende seguir avanzando en esta investigación, con un enfoque cualitativo, desarrollando análisis profundo sobre los stakeholders que intervienen en el branding realizado por algunas de las empresas tradicionales de la ciudad de Manizales. 


\section{Stakeholders}

El concepto stakeholder, que es ampliamente utilizado en casi todos los contextos empresariales, apareció por primera vez en el año 1963 en un memorando interno del Stanford Research Institute (Freeman, Harrison, Wicks, Parmar \& Colle, 2010), y que se definía como los grupos sin los cuales la organización no podría existir. Posteriormente, en el año 1984, el término se popularizó a partir del surgimiento de una publicación de Edward Freeman denominada "Estrategic Mangement: A Stakeholder Approach". Este manuscrito es de vital importancia ya que gracias a él se concibe como cualquier grupo o individuo que puede afectar o puede ser afectado por el logro de los objetivos de la empresa. Además, el eje central de su teoría hará cambiar la manera de entender la relación de la empresa con sus públicos, debido a que justifica que "una empresa no solo es responsable ante sus accionistas y propietarios, sino también ante sus empleados, los consumidores, sus suministradores y el conjunto de los grupos e individuos que son necesarios o que pueden influir en el desarrollo de sus objetivos" (Perdiguero, 2003, p. 160).

Aunque el concepto más utilizado sea el de stakeholder, también se materializa en términos como grupos de interés, partes interesadas, agentes, entre otros. Para Ibisate (2007), se han nombrado de distinta manera según sea su origen, pero son en definitiva la misma idea. También los investigadores de la Asociación Española de Contabilidad y Administración (AECA) (2006) consideran que stakeholders, partes interesadas y grupos de interés "serían, por lo tanto equivalentes, y utilizables de forma sinónima" (p. 34).

Lo interesante de este nuevo enfoque de los stakeholders es que aparecen en escena nuevos públicos, que modifican las estrategias empresariales, ya que se crea la necesidad de entrar en contacto con todos los posibles grupos de interés de la compañía. Estas nuevas partes interesadas serán tan heterogéneas que la empresa deberá multiplicar esfuerzos para cumplir sus responsabilidades, además de crear nuevos modelos de gestión y administración que le permitan mejorar su mutua relación (Lozano, 2008). Ahí radica la importancia de esta perspectiva stakeholder.

Actualmente las investigaciones sobre empresa y marca hacen alusión a este término, debido a que abarca los diferentes grupos de interés, permitiendo de esta forma analizar holísticamente el contexto. En este sentido, la importancia que tienen los stakeholders o públicos de interés para una empresa es considerable, pues no es posible la proyección empresarial sin el capital humano. Estos públicos de interés son los accionistas, ejecutivos, empleados del sector operativo, proveedores, consumidores, competencia, autoridades gubernamentales, periodistas y, como se mencionó anteriormente, todas las personas que puedan ser afectadas o afecten el desarrollo de la empresa; es de aclarar que cada empresa define sus stakeholders.

La forma de clasificar los tipos de stakeholders puede ser muy variada, dependiendo no solo del enfoque del investigador, sino también de situaciones prácticas como tipo de empresa, tamaño, sector al que pertenece, amplitud de la cadena de valor, entre otros factores. Para Araque \& Montero (2006), ya que no existe un acuerdo unánime de cómo 
clasificar los stakeholders, se debe entender cómo se concibe el contenido y los límites de la RSC. Por consiguiente, las posibilidades de clasificación de los stakeholders se pueden enunciar según la propuesta de sus autores (tabla 1).

Tabla 1. Clasificación de los stakeholders

\begin{tabular}{|c|c|}
\hline Autor & Clasificación \\
\hline Dowling (2001) & $\begin{array}{l}\text { - } \quad \text { Normativos: directivos, accionistas, entes reguladores, gobierno. } \\
\text { - } \text { - Duncionales: empleados, sindicato, proveedores, distribuidores. } \\
\text { - } \text { Consumidores: segmentos por necesidades. }\end{array}$ \\
\hline Ibisate (2007) & $\begin{array}{l}\text { - Estructurales: propietarios, accionistas, inversores y socios. } \\
\text { - Gestión: empleados, clientes, proveedores, reguladores, entidades financieras, usuarios finales. } \\
\text { - Complementarios: administraciones, comunidades locales, ONG, organizaciones generadoras } \\
\text { o creadoras de opinión, medios de comunicación. }\end{array}$ \\
\hline Navarro (2008) & $\begin{array}{l}\text { - Internos: accionistas, socios, directivos, sindicatos, trabajadores, socios estratégicos. } \\
\text { - Externos: autoridades, grupos de presión, ONG, competidores, consumidores. }\end{array}$ \\
\hline Azuero (2009) & $\begin{array}{l}\text { - Los que perderían algo si la empresa cerrara: trabajadores y sus familias, consumidores, pro- } \\
\text { veedores, administraciones locales, inversores. } \\
\text { - Los que pueden influir en mi marca: empleados y consumidores, medios de comunicación, } \\
\text { ONG, agencias reguladoras, la competencia. } \\
\text { - Todos los grupos que representan intereses que pueden ser afectados por la operación de la } \\
\text { empresa: grupos ecologistas, asociaciones cívicas, grupos de presión, gobiernos. }\end{array}$ \\
\hline $\begin{array}{l}\text { Raghubir, Ro- } \\
\text { berts, Lemon } \\
\text { E Winer (2010) }\end{array}$ & $\begin{array}{l}\text { - Estratégicos: consumidores, proveedores, competidores, empresa. } \\
\text { - Internos: inversores, empleados, directivos. } \\
\text { - Externos: sociedad, medio ambiente, entes reguladores medios de comunicación, mercados, } \\
\text { economía. }\end{array}$ \\
\hline
\end{tabular}

Fu <ente: elaboración propia (orden por fecha)

Las diferentes tipologías de los stakeholders deberán ser asumidas directamente por cada empresa, dependiendo de las circunstancias de los mercados, del contexto, de los productos, etc. Además, cambiará la relación de estos con la empresa, pues no serán los mismos stakeholders los de una empresa multinacional con representación en varios continentes, que los de una empresa con presencia local. Las implicaciones legales, financieras, económicas y políticas de cada una serán de alto contraste; de allí la importancia de que la propia compañía haga el esfuerzo para caracterizar sus stakeholders.

Es por esto que la comunicación de una empresa, a través de sus intangibles -el sistema de la marca-, debe ser muy clara y estructurada, con el fin de situarse de manera firme en la mente de todos los stakeholders (Chaves, 2008; Ávalos, 2010). Solo de esta 
forma es posible generar imagen y reputación de marca positivas, intangibles clave en la estructura de una empresa (Villafañe, 2009; López \& Iglesias, 2010; Orozco \& Ferré, 2012). Por ello las empresas realizan importantes inversiones en la comunicación estratégica de la marca -branding-, incluyendo los diferentes grupos de interés, con el fin de estructurar una imagen de marca coherente con la identidad que se desea proyectar (Costa, 2004; Aaker \& Joachimsthaler, 2005; García, 2005; Capriotti, 2009).

Para Orozco y Ferré (2012) los stakeholders o grupos de interés son determinantes en la comunicación estratégica de las empresas, más aún en esta época donde las tecnologías de la información y la comunicación (TIC) están al alcance de cualquier persona. A través de las TIC los stakeholders pueden divulgar el buen o mal desempeño que tiene una empresa en cuanto a su relación con el medio ambiente, la sostenibilidad, la cultura, entre otros sectores de la sociedad.

A partir de esta teoría, las decisiones empresariales asumen a los stakeholders como interlocutores. Aunque en este aspecto las TIC han desempeñado un papel importante, las empresas son cada vez más cuidadosas con las comunicaciones que realizan con sus grupos de interés (Durán, 2005). Por ejemplo, en el caso de la web 2.0, Fuster \& Ortega (2010) manifiestan que cualquier usuario que tenga una conexión a Internet puede ser una fuente potencial de escándalo corporativo; de allí la importancia del diálogo con los stakeholders.

La importancia de la teoría de los stakeholders no solo se basa en la perspectiva de la empresa, pues así se genere gran variedad de impactos para la organización, los directamente beneficiados no son unos pocos grupos de interés, sino públicos amplios, diversos, con los que incluso existen intereses contradictorios (Polo, 2011). La organización es también consciente de la importancia de la totalidad de su cadena de valor (Peinado, 2011), lo que permite no dejar por fuera del proceso a ningún grupo de afectados, y así tratar de buscar siempre la satisfacción de múltiples stakeholders (Freeman, Harrison $\mathcal{E}$ Wicks, 2007).

Por otra parte, la relación con los stakeholders permite conocer la forma como debe actuar la empresa en un contexto determinado. Sin embargo, no es suficiente solo con conocer las características de los diferentes grupos de interés; adicional a esto la empresa debe asumir un rol que promueva la participación colectiva, es decir, descentralizar su forma de actuar en el medio. Esto quiere decir que la empresa debe tener en cuenta a los stakeholders en sus decisiones y sus acciones estratégicas, ya sean estas en el ámbito de la proyección de sus tangibles o en la comunicación estratégica de sus intangibles.

Al respecto Freeman (1994) considera que las acciones y políticas de las empresas deben estar mediadas por los intereses de los diferentes stakeholders; sin embargo, estas acciones deben ser equilibradas, debido a que los intereses pueden ser contradictorios, y algunos pueden ir en contra de los beneficios de la empresa. Es importante, según Freeman (1994), ceder a ciertas exigencias de los stakeholders de manera equilibrada, pero no solo basta con tener en cuenta los grupos de interés como los accionistas y consumidores, 
los cuales afectan de manera más directa a la empresa, sino que es importante incluir a otros grupos como empleados, proveedores y comunidad en general.

Cada grupo de stakeholders asume un rol diferente, incidiendo directa o indirectamente en la empresa; por esta razón, el éxito de una empresa depende, en gran parte, de la forma como se gestionen las relaciones con los diferentes grupos de interés (Clarkson, 1995; Capriotti, 2013). En este orden de ideas, el diálogo con todos los stakeholders debe hacerse continuamente, identificando las características y necesidades de cada uno de estos grupos, con el fin de tener bases suficientes para la comunicación y proyección estratégica de la empresa, es decir, aquellas actividades inherentes al branding y la gerencia.

Finalmente, es importante añadir que los stakeholders han hecho que las empresas deban pensar en todos los públicos, no solo desde la perspectiva de la comunicación, sino también en todo lo relacionado con el branding y los demás aspectos de la marca.

\section{Stakeholders e identidad de marca}

La misión y la visión son ejes clave en la construcción de la identidad de marca, por lo cual, se debe tener muy claro hacia dónde quiere ir la empresa, sus objetivos y enfoque en la relación con los diferentes stakeholders. No es posible construir una identidad bien estructurada sin tener en cuenta estos aspectos, pues la comunicación de valores o atributos, a través del sistema identitario, debe estar soportada por la razón de ser de la empresa. Es por esto que la identidad de cualquier empresa, en sus diferentes dimensiones, debe tener una estrecha relación con su quehacer y obviamente con sus grupos de interés (Sanz de la Tajada, 1994; Chaves, 2008).

En la construcción del sistema identitario inciden directa o indirectamente diferentes stakeholders. Inicialmente, para que la identidad sea concebida adecuadamente se debe tener en cuenta el target o grupo de consumidores potenciales, con el fin de generar conexión y, de esta forma, influir sobre la decisión de compra. De otro lado, participa directamente, en la construcción de identidad, un grupo creativo que está conformado por stakeholders, donde se encuentran profesionales de diferentes áreas; de igual forma, otros stakeholders como empleados del sector ejecutivo, accionistas, asesores, entre otros. Este proceso de construcción de identidad se da paulatinamente y se refuerza constantemente, de manera que vaya adquiriendo cada vez mayor fuerza.

De acuerdo con lo anterior, la identidad de marca es un proceso endógeno y exógeno que se da de forma iterativa, es decir, las acciones internas repercuten en la sociedad, y las dinámicas sociales obligan a las empresas a modificar su estructura identitaria y comunicativa (Toffler, 1990; Gázquez, \& Sánchez, 2004; Bauman, 2010). Es así como se da una constante interacción entre los stakeholders internos y externos, lo cual afecta directa o indirectamente la identidad de marca de cualquier empresa.

Las empresas deben estar atentas a lo que sucede en el entorno donde interactúan sus stakeholders, debido a que los cambios que se puedan dar son señales que indican la 
necesidad de modificar los productos, los servicios, la estrategia de comunicación o cualquier otro ámbito relacionado con la empresa, según sea el caso. Los posibles cambios del entorno se pueden detectar a través de la investigación y el análisis de lo que sucede con las tendencias, los públicos objetivo y en general los diferentes grupos de interés, así como la competencia misma.

Los stakeholders son personas, y como tal son susceptibles de cambiar, por lo cual algunas empresas deciden cambiar su estructura identitaria -asunto que se recomienda hacer con mesura-, con el fin de renovar su relación con los grupos de interés o para estar a la vanguardia con las tendencias o el estilo de la época. De este modo es como la identidad de marca está condicionada a la relación con los stakeholders, pues, finalmente, la relación con los grupos de interés condiciona en gran medida el éxito o fracaso de la empresa.

De acuerdo con lo anterior, es importante que la identidad de marca esté estrechamente relacionada con los stakeholders, pues solo de esta forma es posible consolidar un sistema identitario con el cual se sientan identificados los grupos de interés, logrando así vínculos que puedan perdurar a través del tiempo. Esto hace parte de los valores intangibles de una empresa y permite establecer una ruta clara en la construcción de marca, asunto que es clave en la comunicación estratégica de cualquier organización con los stakeholders.

\section{Stakeholders, imagen y reputación de marca}

La imagen de marca se forma en la mente de los stakeholders a lo largo del tiempo. Es un proceso que se va dando paulatinamente (no se da de forma instantánea) y depende de las acciones de la empresa a través de la gestión de sus tangibles e intangibles y su interacción con el medio (Costa, 2001; Aaker \& Joachimsthaler, 2005; García, 2005; Chaves, 2008; Capriotti, 2013). De acuerdo con esto, la imagen es la percepción de los valores de marca, los cuales son susceptibles de cambiar en cualquier momento, por lo cual las empresas deben tener mucho cuidado con sus estrategias de comunicación aplicadas en el branding y su repercusión en la mente de los públicos de interés. Sin embargo, no es posible controlar la imagen de marca, es decir, la percepción que tienen los grupos de interés sobre la empresa, pero sí es posible realizar un seguimiento sobre la forma como perciben la marca los diferentes stakeholders.

Para Orozco \& Roca (2011) "la imagen será una percepción subjetiva, ya que será el resultado de una gran cantidad de inputs que recibe el sujeto durante su "experiencia" con la marca" (p. 275); todo esto se puede traducir en valores de marca que se forman desde el interior de la empresa o desde el exterior de la misma.

La imagen de marca que tienen los empleados del sector operativo, ejecutivos e inversionistas puede ser muy cercana entre ellos, sin embargo, puede no ser tan cercana a la imagen de marca que tienen los consumidores, los proveedores y los demás públicos externos. A pesar de esto, es importante recordar que la imagen de marca, en general, de todos los grupos de interés debe ser coherente con la identidad de marca, es decir, debe haber poca brecha entre identidad e imagen. Esto solo es posible lograrlo con una 
construcción estratégica de marca - branding-adecuada, a través de estudios de mercado, diálogo con los diferentes públicos de interés, campañas publicitarias, diseño de sistemas de identidad, la RSE, entre otras acciones.

La proliferación de productos, marcas, medios, etc., ha generado una saturación inmensurable de mensajes que no permiten que la empresa pueda transmitir una comunicación unificada que estreche las diferencias entre la identidad y la imagen corporativa de los sujetos. Esta es una de las razones por las que la comunicación entre la empresa y los stakeholders es tan importante.

De otro lado, cuando se menciona el concepto "imagen" es necesario referirse al término "reputación"; ambos están relacionados con el ámbito corporativo y debido a que tienen estrecha relación suelen confundirse o abordarse indiscriminadamente. Para Villafañe (2009) la reputación es la consolidación de la imagen a través del tiempo; este término tiene más relación con el posicionamiento de la empresa, mientras que la imagen solo representa la percepción de atributos; el primer concepto, para el autor, tiene mayor relación con la identidad corporativa, y el segundo concepto está más relacionado con la personalidad corporativa. De acuerdo con esto la reputación representa un conocimiento más profundo sobre la empresa; sin embargo, esto no quiere decir que la imagen no implique conocimiento sobre la misma, por lo cual, es difícil saber hasta qué punto la percepción de los stakeholders implique reputación o imagen.

En relación con Villafañe (2009), Orozco \& Ferré (2012) aducen que la reputación es un conocimiento más profundo que la imagen, es decir, "[...] va más allá, hasta el conocimiento de aspectos tan concretos como los resultados financieros, los comportamientos corporativos e incluso las labores de responsabilidad corporativa de una empresa" (p. 62). De esta forma es como se plantea la diferencia entre reputación e imagen, donde hay que resaltar el valor que tiene el tiempo y la reiteración en la mente de los stakeholders, es decir, cómo la comunicación estratégica y reiterativa de los valores de marca, con el pasar del tiempo, va generando una especie de impronta en la mente de los grupos de interés.

En este sentido se entiende que el vínculo entre los públicos de interés y la reputación es tan fuerte que el mismo Costa (2009) precisa que la reivindicación de la reputación en los últimos años ha sido originada por la irrupción de la noción de grupos de interés, ya que se ha ampliado el punto de mira sobre el que las empresas deben tener presente su diario accionar. Aunque Caruana (2008) revele que la RC es el resultado de las percepciones de todos los stakeholders, se debe considerar oportuno investigar y definir cuáles son las percepciones de cada uno de los grupos de interés de la compañía.

Según sea el caso, una misma marca puede tener diferentes reputaciones dependiendo de las percepciones de sus stakeholders. Esta es la razón por la cual se considera la reputación como un valor difícil de controlar, debido a que "it is the publics that build reputation, not organizations themselves" (Aula \& Mantere, 2008, p. 210).

Dada la evidente dificultad de gestionar la relación con diversos grupos de interés, los 
directivos, y en especial los encargados del diseño de la identidad y de la comunicación, deben dominar lo que Villafañe (2004) llama el diálogo multistakeholder. Es decir, controlar los mensajes para que exista equivalencia entre lo que la organización quiere dar a conocer a través de la comunicación de su identidad y las percepciones de los stakeholders a través de la imagen y la reputación.

Por su parte Capriotti (2009) relaciona un término adicional -posicionamiento- a la disyuntiva entre reputación e imagen. Sin embargo, el autor plantea que no debería haber diferenciación entre los tres conceptos -imagen, reputación y posicionamiento-, debido a que todo es asunto de emisores -empresas-y receptores -públicos-. En relación con Capriotti (2013), para el estudio sobre los stakeholders, es posible que la diferenciación de dichos conceptos no ayude mucho a la hora de realizar estudios sobre las percepciones de los diferentes grupos de interés, debido a que pueden ser tan valiosas las percepciones que tengan los stakeholders con poco conocimiento sobre la empresa, como la de los stakeholders con mayor conocimiento sobre la misma.

\section{Stakeholders, posicionamiento y goodwill}

La identidad de marca y su relación con las acciones de la empresa incide en la construcción de imagen formada en la mente de los stakeholders, lo cual lleva, con el pasar del tiempo, a la generación de reputación corporativa. Luego la reputación, generada a través de la imagen de marca y su relación con los resultados de la empresa, conduce a la conformación del posicionamiento, concepto que se refiere al escalón que ocupa la marca, en relación con otras de la misma categoría, en la mente de cualquier persona (Ries, Trout \& Ampudia, 1982; Hatch \& Schultz, 2000; Keller, 2008; Fernández, 2011). En esta dirección es posible considerar que la posición en los primeros escalones depende de la calidad del producto o servicio, el branding, la adaptación a nuevos mercados, entre otros aspectos.

En este sentido se concibe que "el enfoque fundamental del posicionamiento no es crear algo nuevo y diferente, sino manipular lo que ya está en la mente; re-vincular las conexiones que ya existen" (Ries \& Trout, 1992, p. 5). Por ello, se deben conocer muy bien los stakeholders, para así focalizar los esfuerzos de la empresa en una buena comunicación con los grupos de interés.

Es importante que durante este camino, se tenga una consciencia frente a lo que ya existe en la mente de los públicos de interés y lo que implica pelear por un primer escalón, en caso de que ya esté tomado por otro producto. Asimismo, los beneficios que trae determinar el "segundo" escalón como objetivo: "muchas empresas caen en un error que consiste en confundir el posicionamiento deseado (el que quieren conseguir o desean) con el posicionamiento real (el que otorga el consumidor)" (Llopis, 2015, p. 179).

La comunicación adecuada con los diferentes stakeholders es clave en el posicionamiento de las marcas, debido a que en la actualidad algunas personas tienen a la mano herramientas de comunicación que pueden utilizar en contra o a favor de un producto (Clarkson, 1995; Ros \& Castelló, 2011; Capriotti, 2013). Por esta razón es necesario que los 
encargados de las estrategias de comunicación estén atentos a cualquier hecho que se pueda presentar en redes sociales, medios de comunicación o incluso el voz a voz, que en algunas ocasiones puede ser aún más influyente. Esto reafirma la importancia que tienen los intangibles de una empresa; incluso, las marcas más importantes del mundo se caracterizan por que el valor de sus intangibles -marca- supera considerablemente el valor de sus activos. Así se evidencia en el informe sobre índices bursátiles "Standard $\varepsilon$ Poor's 500", el cual concluye que "en los treinta años transcurridos entre 1975 y 2005, la contribución de los activos intangibles al valor global de las empresas había aumentado de un 17 a un $80 \% "$ (Lindemann, 2010, p. 50).

Estos intangibles de la marca representan lo que se conoce como "goodwill", término directamente relacionado con la reputación corporativa (Salinas, 2007; Atta, 2012). Para Salinas (2007) el goodwill es la diferencia entre el valor de mercado y el valor libros. Es decir, entre mayor sea el valor intangible -la marca-, en relación con los activos reales de la empresa, mayor será la reputación o más importante será el goodwill. Este término comenzó a utilizarse cuando la marca, como valor intangible, empezó a superar el valor real de la empresa; aspecto característico de la Posmodernidad, donde la imagen se convierte en un elemento de gran influencia en la comunicación, la interacción social y el comportamiento humano (Castells, 2009; Bauman, 2010; Mitchell, 2010).

Bajo estas condiciones, el goodwill no puede ser reemplazado ni equiparado al de reputación, pues es un concepto que tiene sus raíces en la contabilidad, pero sigue teniendo una fuerte influencia con respecto a la relación que tiene la empresa con cada uno de sus stakeholders. Así las cosas, el goodwill será más relevante en la medida en que la empresa sea más importante y tenga una mayor significación para los grupos de interés.

\section{Stakeholders y marketing experiencial}

A través del marketing es posible conocer las características de los consumidores y los diferentes grupos de interés, debido a que esta disciplina ofrece herramientas útiles para el conocimiento de las dinámicas comerciales, mediante la investigación del mercado; esto puede aportar en la construcción de relaciones más estrechas entre los stakeholders y la marca. Para Kotler \& Armstrong (2003) "el marketing es la función comercial que identifica las necesidades y los deseos de los clientes, determina qué mercados meta puede atender mejor la organización y diseña productos, servicios y programas apropiados para atender esos mercados" (p. 19). De acuerdo con esta definición, el branding requiere de los asuntos inherentes al marketing, pues el conocimiento de las características del mercado determina lineamientos y directrices que se deben aplicar en las estrategias de la marca, con el fin de incrementar el valor de los tangibles e intangibles (Semprini, 1995; Aaker $\&$ Joachimsthaler, 2005; García 2005; Keller, 2008; Fernández \& Labarta, 2009).

Es importante que las acciones de marketing no solo se centren en los consumidores y públicos objetivo; abordar los stakeholders, así como los clientes, es clave para construir imagen y reputación consistentes, tanto desde afuera como en el interior de la empresa. 
Bajo esta premisa, Schmitt (2000) propone el marketing experiencial como una forma de crear recordación positiva en los clientes, generando experiencias que permitan hacer asociaciones y recuerdos que refuercen la dimensión emocional, para producir, de esta forma, un valor diferencial en la marca.

Esto debe realizarse extendiendo las acciones desde y hacia el interior de la empresa, integrando los stakeholders internos a través de la relación con el cliente, la iluminación, el aroma, entre otros elementos relacionados con el punto de venta o los demás espacios que impliquen interacción entre las personas. Bajo estas nuevas premisas, Aguilera $\varepsilon$ Baños (2016), advierten que:

Un negocio en el que los clientes pagan por el tiempo en que se ha estado con la marca es un negocio de experiencias, al que diferencian de otros, como el de los commodities, en el que se paga por el material, el de los bienes, en el que se paga por objetos tangibles, o el de los servicios, en el que se paga por las actividades que desempeña la marca. Cada vez más, el marketing es experiencial, no solo se dedica a comunicar ventajas y beneficios de productos, sino que también conecta una determinada oferta con experiencias únicas e interesantes. Por lo tanto, el objetivo no es vender algo, sino demostrar la forma con la que una marca puede enriquecer la vida de un cliente (Aguilera \& Baños, 2016, p. 71).

Esta visión permite entender que el marketing y su relación con los grupos de interés hacen parte de los aspectos relacionados con la construcción de marca o branding, asunto que también se ha abordado desde el diseño experiencial, con el fin de generar conexiones más estrechas entre los stakeholders y la marca (Press \& Cooper, 2009).

Desde la perspectiva de la publicidad, se han realizado esfuerzos en la gestión de experiencias enfocadas en los grupos de interés, en especial en la creación de contenidos divertidos y únicos, pues es la forma en que "en lugar de interrumpir a los consumidores con anuncios, se trata de atraerlos, de engancharlos (engage), con utilidad, entretenimiento, información relevante o cualquier otro tipo de contenido valioso que sea verdaderamente bien acogido por el consumidor" (Aguilera \& Baños, 2016, p. 102). Estas nuevas prácticas empresariales lo que permiten es dar cuenta de la necesidad que tienen las marcas de relacionarse con los stakeholders a partir de estrategias de comunicación diseñadas para mejorar la experiencia de los clientes y la confianza de ellos hacia la organización.

\section{Conclusiones}

Los stakeholders o grupos de interés son parte integral de las empresas, sin ellos no es posible la existencia de las mismas. Por esta razón las empresas deben gestionar sus intangibles cuidadosamente, buscando la mejor forma de conectarse con sus stakeholders. Solo de esta forma es posible construir una imagen positiva en los diferentes grupos de interés, lo cual se traduce en credibilidad, confianza, reconocimiento, entre otros atributos que fortalecen la marca y la empresa.

Las investigaciones sobre los stakeholders han permitido a las marcas proyectarse de 
una manera más precisa, debido a que el conocimiento de los diferentes actores, que participan directa o indirectamente en el desarrollo de la empresa ofrece herramientas útiles para la comunicación estratégica de marca -branding-. Es por esto que las grandes marcas invierten en investigaciones enfocadas en estudiar el comportamiento de los consumidores y otros grupos de interés, con el fin de obtener información útil para la construcción de estrategias dirigidas a incrementar el valor de sus tangibles e intangibles.

A través de los grupos de interés es posible diagnosticar la imagen o la reputación de marca, y de esta forma revisar qué aspectos se deben mejorar en la identidad y en los demás elementos que hacen parte de la comunicación de la empresa. Los eventuales cambios que se hagan, sobre todo en la parte de identidad de marca, deben realizarse con mesura, de manera que no se generen distorsiones en la imagen de la empresa. Otros posibles cambios pueden efectuarse en el ámbito de las acciones estratégicas, donde se involucre a los stakeholders, asunto que debe manejarse equilibradamente, de manera que los beneficios sean tanto para los públicos de interés como para la empresa.

La imagen y la reputación de marca se construyen de manera paulatina y son el resultado de la comunicación de la empresa con sus stakeholders. De esto depende, en gran medida, el posicionamiento que tengan la marca o empresa en el entorno, asunto que en la actualidad determina el éxito o fracaso empresarial.

Aunque la imagen y la reputación varían de acuerdo con cada grupo de stakeholders, la idea es que sean cercanas a la identidad; por esto las estrategias deben enfocarse en disminuir la brecha entre identidad de marca e imagen o reputación de marca. Esto puede lograrse manteniendo estrategias de comunicación adecuadas con los diferentes grupos de interés; además, algunas empresas realizan acciones adicionales en el ámbito del marketing experiencial, con el fin de extender y complementar sus relaciones con los grupos de interés.

\section{Referencias}

Aaker, D. E Joachimsthaler, E. (2005). Liderazgo de la marca. Barcelona, España: Deusto.

Aguilera, J. \& Baños, M. (2016). Branded entertainment. Madrid: ESIC.

Araque, R. E Montero, M. (2006). La responsabilidad social de la empresa a debate. Barcelona: Icaria.

AECA (2006). La semántica de la responsabilidad social corporativa. Madrid: AECA.

Aula, P. E Mantere, S. (2008). Strategic reputation management. Nueva York: Routledge.

Atta, S. (2012). Everything good will come. Massachusetts: Interlink Publishing.

Ávalos, C. (2010). La marca. Identidad y estrategia. Buenos Aires: La Crujía.

Azuero, D. (2009). La comunicación de la RSE. Propuestas para un modelo de comunicación responsable. Madrid: Forética.

Bauman, Z. (2010). Mundo consumo. Ética del individuo en la aldea global. Barcelona: Paidós. 
Capriotti, P. (2009). Branding corporativo: Fundamentos para la gestión estratégica de la identidad corporativa. Santiago de Chile: Colección de Libros de la Empresa.

Capriotti, P. (2013). Planificación estratégica de la imagen corporativa. Málaga: Instituto de Investigación en Relaciones Públicas.

Caruana, A. (2008). An attitudinal measure of corporate reputation. En Melewar, T. (Ed.), Facets of corporate identity, communication and reputation (pp. 197-209). New York: Routledge.

Castells, M. (2009). Comunicación y poder. Madrid: Grupo Anaya Comercial.

Clarkson, M. (1995). A stakeholder framework for analyzing and evaluating corporate social performance. Academy of Management Review, 20(1), 92-117.

Costa, J. (2001). Imagen corporativa en el siglo XXI. Buenos Aires: La Crujia Ediciones.

Costa, J. (2004). DirCom on-line. La Paz, Bolivia: Grupo Editorial Design.

Costa, J. (2009). El DirCom hoy. Dirección y gestión de la comunicación en la nueva economía. Barcelona: Costa Punto Com.

Chaves, N. (2008). La imagen corporativa. Teoría y práctica de la identificación institucional. Barcelona: Gustavo Gili.

Dowling, G. (2001). Creating corporate reputations. Identity, image, and performance. Nueva York: Oxford University Press.

Durán, J. (2005). La empresa multinacional. Gobernanza y responsabilidad social. Madrid: Cámara Madrid.

Fernández, J. E Labarta, F. (2009). Cómo crear una marca. Manual de uso y gestión. Córdoba: Almuzara.

Fernández, M. (2011). Comunicación y reputación en empresas e instituciones. Experiencias profesionales y propuestas prácticas. Madrid: Universitas S. A.

Freeman, E., Harrison, J. E Wicks, A. (2007). Managing for stakeholders. Survival, reputation and success. New Haven: Caravan.

Freeman, E., Harrison, J., Wicks, A., Parmar, B. E Colle, S. (2010). Stakeholder theory. The state of the art. Cambridge: Cambridge University Press.

Freeman, R. (1994). The politics of stakeholders theory: some future directions, Business Ethics Quarterly, 4 (1), 409-421.

Fuster, A. E Ortega, A. (2010). La RSE en transición. En Morata, F., Vilá, B. E Suárez, C. (Eds.), La responsabilidad social de la empresa a debate. Lecciones de la crisis (pp. 92-101). Barcelona: IUEE.

García, M. (2005). Arquitectura de marcas: Modelo general de la construcción de marcas y gestión de sus activos. Madrid: ESIC.

Gázquez, J. E Sánchez, M. (2004). La identidad e imagen de marca. En Jiménez, A. (Ed.), Dirección de productos y marcas (pp. 56-82). Barcelona: UOC.

Hatch, M. \& Schultz, M. (2000). Scaling the tower of Babel: relational differences between identity, image, and culture in organizations. En Schultz, M., Hatch, M. E Larsen, M. (Eds.), The expressive organization. Linking identity, reputation, and the corporate brand (pp. 11-35). Nueva York: Oxford University Press. 
Ibisate, A. (2007). La gestión corporativa de los grupos de interés. En Real Academia de Ciencias Económicas y Financieras. (Eds.), La responsabilidad social de la empresa. Propuesta para una economía de la empresa responsable y sostenible (pp. 281-287). Barcelona: Racef.

Keller, K. (2008). Administración estratégica de marca branding. México: Pearson.

Kotler, P. E Armstrong, G. (2003). Fundamentos de marketing. México: Pearson.

Lindemann, J. (2010). El valor financiero de la marca. En Brujó, G. (Ed.), En clave de marcas (pp. 48-65). Madrid: LID.

Llopis, E. (2015). Crear la marca global. Modelo práctico de creación e internacionalización de marcas. Madrid: ESIC.

López, V. E Iglesias, S. (2010). Reputación y rendimiento sostenible en pymes. Revista Europea de Dirección y Economía de la Empresa, 19(1), 87-106.

Lozano, J. (2008). CSR or RSC? (Beyond the Humpty Dumpty syndrome). Society and Business Review, 3(3), 191-206.

Mitchell, W. (2010). Teoría de la Imagen. Ensayos sobre representación verbal y visual. Madrid: Akal.

Navarro, F. (2008). Responsabilidad social corporativa: teoría y práctica. Madrid: ESIC.

Orozco, J. E Ferré C. (2012). El ADN de la marca: La concepción de sus valores intangibles en un contexto dialogado. Signo y Pensamiento, 31(61), 56-71.

Orozco, J. E Roca, D. (2011). Construcción de imagen de marca y reputación a través de campañas publicitarias de RSC. Sphera Pública, 11(1), 273-289.

Peinado, E. (2011). "RSE, grandes compradores y la cadena de valor". En Vives, A. E Peinado, E. (Eds.), La responsabilidad social de la empresas en América Latina, (pp. 143-161). Washington: Cumpetere.

Perdiguero, T. (2003). La responsabilidad social de las empresas en un mundo global. Barcelona: Anagrama.

Polo, J. (2011). La RSC de las grandes empresas españolas con presencia en América Latina. Madrid: Fragua.

Press, M. y Cooper, R. (2009). El diseño como experiencia. Barcelona: Gustavo Gili, SL.

Raghubir, P., Roberts, J., Lemon, K. E Winer, R. (2010). Why, when, and how should the effect of marketing be measured? A stakeholder perspective for corporate social responsibility metrics. Journal of Public Policy E Marketing, 29(1), 66-77.

Ries, A., Trout, J., \& Ampudia, G. P. (1982). Posicionamiento. México: McGraw Hill.

Ries, A. E Trout, J. (1992). Posicionamiento. México: McGraw Hill.

Ros, V., E Castelló, A. (2011). La comunicación de la responsabilidad en los medios sociales. Revista Latina de Comunicación Social, 67, 47-67.

Salinas, G. (2007). Valoración de marcas. Bilbao: Deusto. Sanz de la Tajada, L. (1994). Integración de la identidad y la imagen de la empresa. Madrid: ESIC.

Schmitt, B. (2000). Experiential marketing, Barcelona España: Bernd H. Schmitt.

Semprini, A. (1995). El marketing de la marca. Una aproximación semiótica. Barcelona: Paidós.

Toffler, A. (1990). La tercera ola. Barcelona: Plaza y Janés.

Villafañe, J. (2004). El estado de la publicidad y el corporate en España y Latinoamérica. Madrid: Pirámide.

Villafañe, J. (2009). Reputación corporativa y RSC. Bases empíricas para un análisis. Telos, 79, 7582. 